\title{
Saccadic Interception of a Moving Visual Target after a Spatiotemporal Perturbation
}

\author{
Jérome Fleuriet and Laurent Goffart \\ Institut de Neurosciences Cognitives de la Méditerranée, UMR 6193, Centre National de la Recherche Scientifique, Aix-Marseille Université, 13331 Marseille \\ Cedex 03, France
}

\begin{abstract}
Animals can make saccadic eye movements to intercept a moving object at the right place and time. Such interceptive saccades indicate that, despite variable sensorimotor delays, the brain is able to estimate the current spatiotemporal ( hic et nunc) coordinates of a target at saccade end. The present work further tests the robustness of this estimate in the monkey when a change in eye position and a delay are experimentally added before the onset of the saccade and in the absence of visual feedback. These perturbations are induced by brief microstimulation in the deep superior colliculus (dSC). When the microstimulation moves the eyes in the direction opposite to the target motion, a correction saccade brings gaze back on the target path or very near. When it moves the eye in the same direction, the performance is more variable and depends on the stimulated sites. Saccades fall ahead of the target with an error that increases when the stimulation is applied more caudally in the dSC. The numerous cases of compensation indicate that the brain is able to maintain an accurate and robust estimate of the location of the moving target. The inaccuracies observed when stimulating the dSC that encodes the visual field traversed by the target indicate that $\mathrm{dSC}$ microstimulation can interfere with signals encoding the target motion path. The results are discussed within the framework of the dual-drive and the remapping hypotheses.
\end{abstract}

\section{Introduction}

Animals can generate movements to intercept the trajectory of a moving visual object and capture it at the right place and time. This performance is impressive when one considers the distributed nature of signals that encode an object in the brain. Indeed, before the target-evoked retinal activity leads to the recruitment of appropriate muscles, the diverging projections within the visual system and the transmissions of signals through multiple relays with diverse conduction velocities and integration times (Pellionisz and Llinás, 1982; Nowak and Bullier, 1997; Schmolesky et al., 1998) lead to neural signals that are spatially and temporally distributed across several brain regions. Yet the common observation that animals are able to intercept an object at the right place and time suggests that the brain can somehow estimate its current spatiotemporal coordinates (hic et nunc). The build-up of this neural estimate is also very rapid since saccadic eye movements can bring the image of a moving target onto the fovea within 150-200 ms (Segraves et al., 1987; Gellman and Carl, 1991; Guan et al., 2005).

A commonly accepted view considers that interceptive saccades are driven by two signals resulting from the recruitment of

\footnotetext{
Received July 29, 2011; revised 0ct. 31, 2011; accepted Nov. 9, 2011

Author contributions: J.F. and L.G. designed research; J.F. and L.G. performed research; J.F. and L.G. analyzed data; J.F. and L.G. wrote the paper.

This work was supported by Agence Nationale de la Recherche (Grant MAPS) and Fondation pour la Recherche Médicale (FDT 20100920165). We thank F. Alexandre, N. Gandhi, M. Goldberg, L. Guerrasio, R. Kalesnykas, P. Legrain, L. Madelain, E. Ryklin, and members of the DyVA team for helpful comments on a previous version, $M$. Martin for animal care, I. Balansard for veterinarian assistance and the reviewers for their constructive comments. This article is dedicated to the memory of Marc Jeannerod.

Correspondence should be addressed to Laurent Goffart Institut de Neurosciences de la Timone, Campus de Sante, $27 \mathrm{Bd}$ Jean Moulin, 13385 Marseille cedex 5, France. E-mail: laurent.goffart@incm.cnrs-mrs.fr.

DOI:10.1523/JNEUROSCI.3896-11.2012

Copyright $\odot 2012$ the authors $\quad 0270-6474 / 12 / 320452-10 \$ 15.00 / 0$
}

independent processing streams. One signal would encode the location where the target is initially detected (snapshot) whereas the other would encode the subsequent target displacement on the basis of target motion signals (Keller and Johnsen, 1990; Gellman and Carl, 1991; Guan et al., 2005; Schreiber et al., 2006). These two signals would then be summed at the level of premotor neurons in the pontomedullary reticular formation (PMRF) to produce the appropriate saccadic motor commands. According to this hypothesis, an accurate saccade requires integrating target motion signals from the time of the snapshot to the saccade end, i.e., during intervals which can be variable. Indeed, if the interception is delayed because of a longer saccade latency or duration, the achievement of accurate saccades implies that the oculomotor system has been informed of the delay added after the snapshot. Moreover, if the saccade is initially misdirected (e.g., because of a blink, the presence of a distracter, or a change in target motion direction), the change in eye position would also have to be taken into account.

In this study, we tested in the monkey this ability to keep track of a target moving in the peripheral visual field when a change in eye position and a delay are unexpectedly added. More specifically, we tested the accuracy of saccades after a brief electrical microstimulation is applied in the deep superior colliculus (dSC) before the saccade onset. The dSC is a brainstem structure at the interface between the sensory systems and the saccade premotor centers in the PMRF (Hall and Moschovakis, 2004; Gandhi and Katnani, 2011). Its microstimulation induces a saccade for which the gaze compensates when applied before saccades toward a static target (Sparks et al., 1983). As will be shown below, saccades can also remain relatively accurate when the microstimulation is applied before intercepting a moving target. 


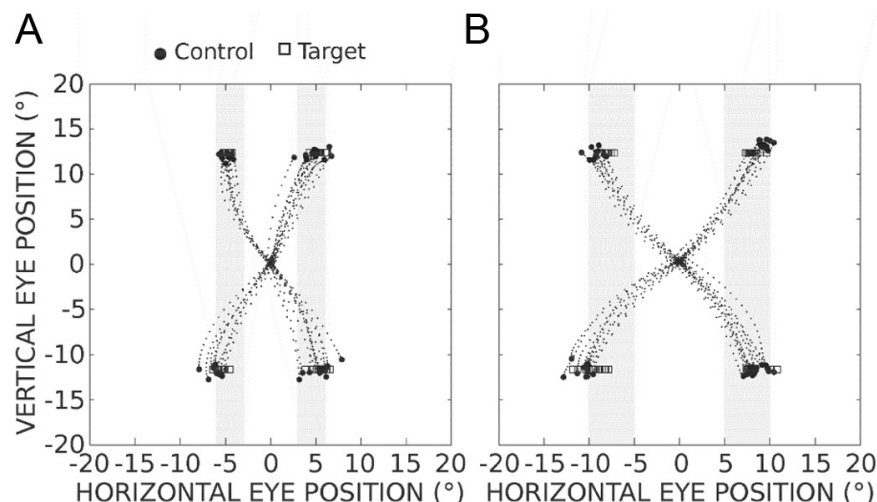

C
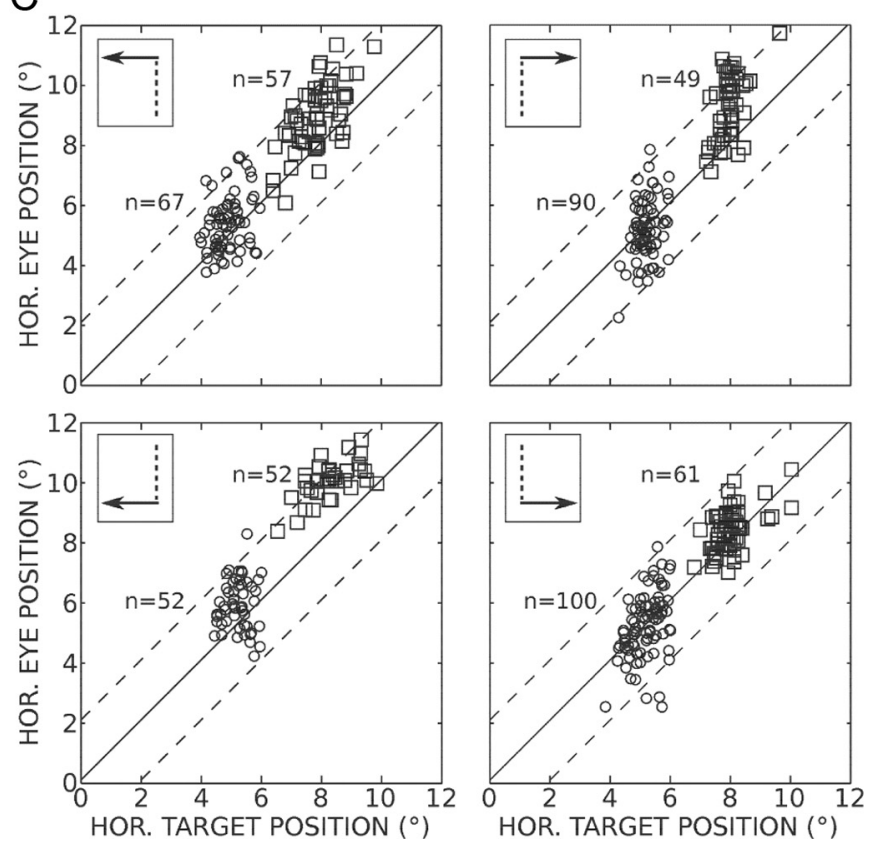

Figure 1. Trajectories and endpoints of control (unperturbed) saccades aimed at a target moving in the periphery. $\boldsymbol{A}, \boldsymbol{B}$, The target steps $12^{\circ}$ in the upper or lower visual field and moves leftward or rightward at a velocity of $20^{\circ} / \mathrm{s}$ (experiment $A 3 ; A$ ) or $33^{\circ} / \mathrm{s}$ (experiment $\mathrm{A} 12 ; \boldsymbol{B}$ ). One hundred fifty milliseconds after the onset of its motion, the target becomes invisible during a blank interval of $150 \mathrm{~ms}$ (gray bars). The saccade endpoints are represented by filled circles. Each open square indicates the location of the target at saccade end. C, The relationship between the horizontal (HOR.) target position and the horizontal eye position (absolute values) at saccade end for each target velocity (circle, $20 \%$; square, $33^{\circ} / \mathrm{s}$ ) for trials where the target moves to the left (left column) or right (right column) after an upward (top row) or downward (bottom row) step (control data from all experiments performed in monkey A). The number of measured saccades is also indicated ( $n$ values).

\section{Materials and Methods}

Subjects and surgical procedures. Two adult male rhesus monkeys (Macaca mulatta, 11-17 kg) were used for this study. Two surgical procedures under isoflurane anesthesia and aseptic conditions were performed. First, a titanium head post, used for the immobilization of the head, was secured to the top front center of the skull using stainless steel screws and bone cement (Gentafix; Teknimed). For the monitoring of eye movements with the magnetic field search coil technique, a threeturn search coil was sutured with silk to the sclera under the conjunctiva of one eye (Fuchs and Robinson, 1966; Judge et al., 1980). Lead wires were passed under the skin to a connector located on the top of the skull. In the second surgical procedure, a craniotomy was performed on the skull, and a recording chamber was stereotaxically positioned for stimulating the dSC. The chamber was centered on the midline and tilted $38^{\circ}$ backward with respect to the frontal plane. The surgical procedures and experiments were performed in accordance with the guidelines from the French Ministry of Agriculture (87/848) and from the European Community (86/609/EEC). Animal care and maintenance were made under the auspices of a full-time veterinarian.
- $20 \%$ s

$\square 33 \%$ s

Eye movement recording and visual stimulation. Data acquisition, on-line control of the oculomotor performance, and triggering of stimuli were controlled by a PC using the Beethoven software package (Ryklin Software). Eye movements were measured with a phase angle detection system (3-feet coil frame; CNC Engineering). Voltage signals separately encoding horizontal and vertical positions of the eye were sampled at $500 \mathrm{~Hz}$, with a resolution of 12 bits (6025e; National Instruments). Monkeys were seated in a chair, facing a video monitor (P227f; $1280 \times 1024$ pixels, $100 \mathrm{~Hz}$ refresh rate, $39 \times 29 \mathrm{~cm}$; ViewSonic Professional Series) located at a viewing distance of $49 \mathrm{~cm}$. Visual stimuli were Gaussian-blurred disks of $0.5^{\circ}$ diameter.

Behavioral task. The animals were trained to perform trials requiring them to orient their gaze from a central target (located in the straight-ahead direction) toward a peripheral one. For each trial, a warning tone preceded the onset of the central target. The monkey's task was to direct its gaze toward it within a surrounding spatial window $\left(2^{\circ}\right.$ radius centered on the target) for a variable interval $(750,1000$, or $1250 \mathrm{~ms}$ ). Following this fixation interval, the central target was extinguished and immediately after (no gap), a peripheral target appeared $12^{\circ}$ along the vertical meridian (downward or upward) and moved with a constant velocity of $20 \%$ s (27 experiments: 12 in monkey A, 15 in monkey M) or 33\% (7 experiments: 4 in monkey A, 3 in monkey $\mathrm{M}$ ) in the horizontal direction (orthogonal to the step), i.e., leftward or rightward. To avoid the use of visual signals for guiding the correction during perturbed trials, the target became invisible $150 \mathrm{~ms}$ after its motion onset and for a blank interval lasting $150 \mathrm{~ms}$ in most experiments (300 ms in 7 experiments: 4 in monkey A, 3 in monkey $\mathrm{M} ; 20 \% \mathrm{~s}$ target velocity). After this blank interval, the moving target reappeared for $500 \mathrm{~ms}$. All possible combinations of target position ( $12^{\circ}$ upward or downward) and motion (leftward or rightward) were applied in pseudorandom order to avoid anticipatory responses. Experiments testing the performance with a target moving at $33 \%$ s or a $300 \mathrm{~ms}$ blank interval were also performed to verify that monkeys did not adopt the strategy of orienting the gaze toward a fixed location in the visual display. The monkeys' task was to generate a saccade toward the target and to pursue it until it reached $16^{\circ}$ of eccentricity (end of trial) within a surrounding spatial window $\left(6^{\circ}\right.$ radius centered on the target). In $50 \%$ of the trials, the interceptive saccade was perturbed before its initiation by an electrical microstimulation in the dSC at the onset of the blank interval (i.e., $150 \mathrm{~ms}$ after the target motion onset). For both unperturbed (control) and perturbed trials, a fruit juice reward was delivered to the monkey at the end of the trial. Depending on the experiment, the number of trials collected per target condition (step and ramp) ranged from 5 to 25 , whether perturbed or not.

Electrical microstimulation. Electrical microstimulation was delivered through a tungsten microelectrode (WE5003; impedance $\sim 0.7-1.2 \mathrm{M} \Omega$, tapered tip; Microprobe) using a stimulus generator (S48; Grass) and photoelectric isolation unit (PSIU6; Grass). During the perturbed trials, electrical microstimulation consisted of trains (duration, $30-45 \mathrm{~ms}$; frequency, $400 \mathrm{~Hz}$ ) of cathodal monopolar pulses ( $0.2 \mathrm{~ms}$ duration). The current (12-20 $\mu \mathrm{A})$ was set to twice the threshold that was found to evoke staircase saccades with a long train $(200 \mathrm{~ms})$ during a separate block of 
stimulation trials. During these trials, the stimulation was applied after extinction of the central target and $200 \mathrm{~ms}$ before the onset of a static target located $10^{\circ}$ on the horizontal or vertical meridians (the monkey was rewarded after fixating this peripheral target). Stimulation was applied only at sites where visuomotor neurons were identified using a delayed saccade task (Mays and Sparks, 1980), 1.2-2 $\mathrm{mm}$ below the surface of the superior colliculus. The vector encoded at the stimulation site was estimated for 31 of 34 sites using optimal stimulation parameters ( 3 times the threshold for evoking contralateral staircase saccades, $200 \mathrm{~ms}, 400 \mathrm{~Hz}$ ).

Data analysis. All data were digitized on-line and analyzed off-line using MATLAB. The onset and offset of the horizontal and vertical saccade components were detected on the basis of a velocity threshold $(30 \%$ ) (for the calculation of eye velocities, see Fleuriet et al., 2011). The results of this automatic detection were checked by inspecting each trial individually and were adjusted manually when necessary. To ensure that all measured saccades were only driven by target signals acquired before the onset of the blank interval, all saccades that ended after this interval were excluded from analysis ( 203 of 743 unperturbed saccades and 126 of 684 perturbed saccades were excluded in monkey A, 271 of 907 unperturbed saccades and 206 of 713 perturbed saccades in monkey $\mathrm{M}$ ). In addition, perturbed trials in which the eye movements displayed one single peak velocity were excluded from analysis because the stimulation-evoked saccade could not be distinguished from the compensatory one (54 movements, 8 sites).

Two parameters, horizontal and vertical final errors, were calculated to estimate the precision and accuracy of the horizontal and vertical components of control and perturbed saccades. The final error was defined as the difference between the eye and target positions at saccade end. By convention, positive errors correspond to hypermetric saccades and negative errors to hypometric ones. Following this, each position value was multiplied by -1 when the target moved leftward. Then, the change in horizontal (vertical) accuracy was estimated by calculating, for each target step and ramp directions, the difference between the median horizontal (vertical) errors of perturbed and unperturbed saccades. A median error was not calculated when $<4$ values were available (because of our exclusion criteria). A nonparametric test (Mann-Whitney test) was performed to compare (within each experiment and for the same directions of target step and ramp) the errors between perturbed and control trials. Finally, the delay that the perturbation added to the time to foveate the target (hereafter called "added delay") was also estimated by calculating the difference between the flight duration (median value) of perturbed saccades and the median duration of unperturbed saccades. For each perturbed saccade, the flight duration is defined as the time interval between the onset of the electrically evoked change in eye position and the end of the correction saccade.

\section{Results}

Figure $1, A$ and $B$, illustrates trajectories of saccades toward a target moving in the upper or lower visual field. In these control trials, after the animal had fixated a central target, a second target appeared on the vertical meridian and moved horizontally toward the left or the right at a constant velocity $[20 \%$ s (Fig. A) or $33 \%$ (Fig. B) ]. To illustrate the fact that the visuosaccadic system can keep track of the position of a moving target in the absence of visual input (Barborica and Ferrera, 2004; Ferrera and Barborica, 2010) and to avoid the use of visual signals for guiding the correction during perturbed trials, the target became invisible 150 $\mathrm{ms}$ after its motion onset and for a blank interval lasting $150 \mathrm{~ms}$ (Fig. 1A, $B$, gray bars). The different landing positions between the graphs in Figure 1, $A$ and $B$, indicate that the saccades were not aimed at a fixed location on the visual display. In fact, the scatter of saccade endpoints during these control trials illustrates the monkeys' ability to estimate the current target location on the basis of retinal signals that were evoked earlier within a very short time window. This extrapolation is further documented in Figure $1 C$, where the horizontal eye position is plotted as a function of the horizontal target position at the time of saccade end (control data from all experiments in monkey A). The two graphs clearly show that the gaze lands beyond the last visible position of the target $\left(3^{\circ}\right.$ horizontally when it moves at $20^{\circ} / \mathrm{s}, \sim 5^{\circ}$ when it moves at $\left.33^{\circ} / \mathrm{s}\right)$. The horizontal position of most saccade endpoints falls within $\pm 2^{\circ}$ of the current horizontal target position.

\section{Distinction between compensations for an ipsilateral and contralateral perturbation}

Figure 2 shows the eye trajectories observed when a brief electrical microstimulation was applied in the dSC at the onset of the blank interval. This microstimulation induced a contralateral eye displacement followed by a correction saccade that oriented the fovea over locations that were close to the target, whether it moved at $20 \%$ s (experiment A3; Fig. $2 \mathrm{~A}$ ) or $33 \%$ (experiment A12; Fig. $2 B$ ). The fact that the electrical stimulation delays the interception is better appreciated in the time course of movements.

Figure 3 shows the changes in horizontal eye position after the onset of the target motion (same experiments as in Figs. $1 A, B, 2$ ). When the microstimulation moved the eye in the direction opposite to the target motion (contralateral perturbation; Fig. $3 A, D)$, the changes in horizontal accuracy are very small $\left(<0.8^{\circ}\right)$ despite the large changes in horizontal eye position [median \pm interquartile range $=5.7 \pm 1.5$ (Fig. $3 A$ ) and $7.6 \pm 1.9^{\circ}($ Fig. $\left.D)\right]$ and longer flight durations $(92 \pm 30$ and $83 \pm 10 \mathrm{~ms}$, respectively) induced by the microstimulation. When the microstimulation moved the eye in the same direction as the target motion (ipsilateral perturbation; Fig. $3 B, E$ ), the correction saccades were slightly less accurate than unperturbed saccades; they often landed on positions located ahead of the target. Regardless of the direction of perturbation, correction saccades were generated with 


\section{$20 \% / \mathrm{s}$}

A
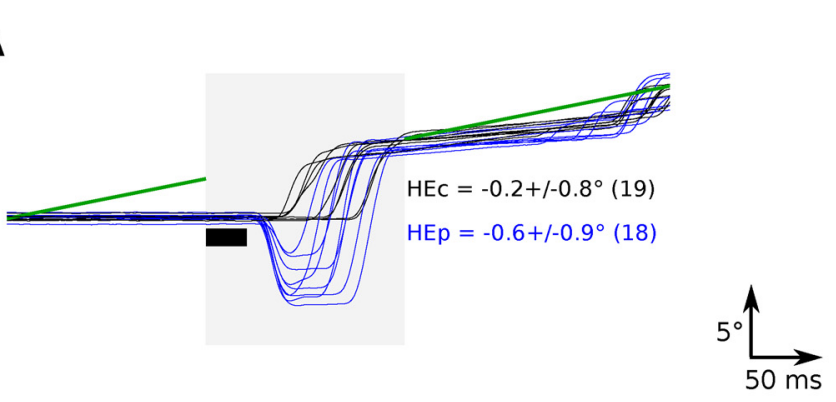

B

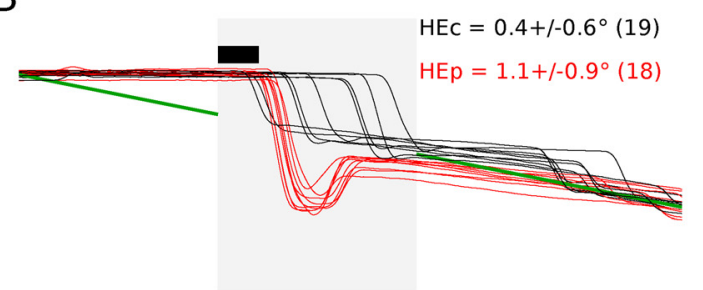

Control

C

HORIZONTAL

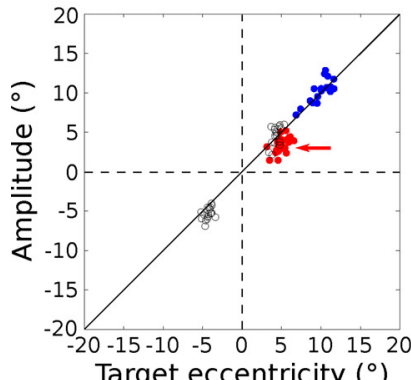

Target eccentricity $\left({ }^{\circ}\right)$
Target

VERTICAL

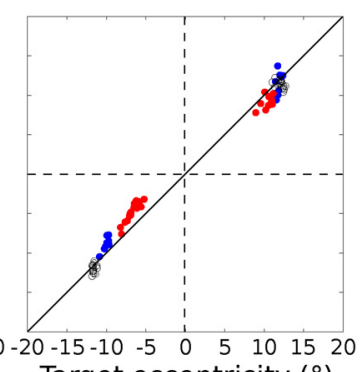

Target eccentricity $\left({ }^{\circ}\right)$
D

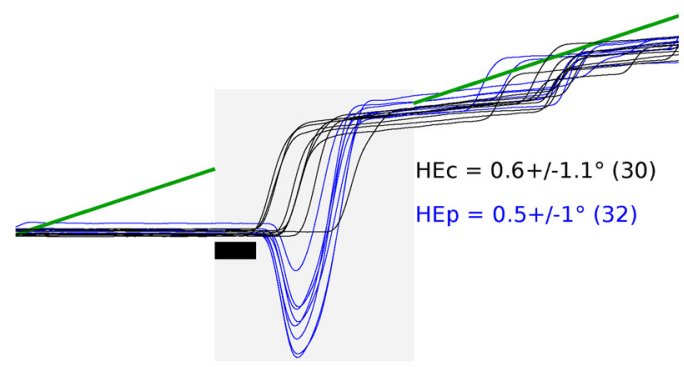

E

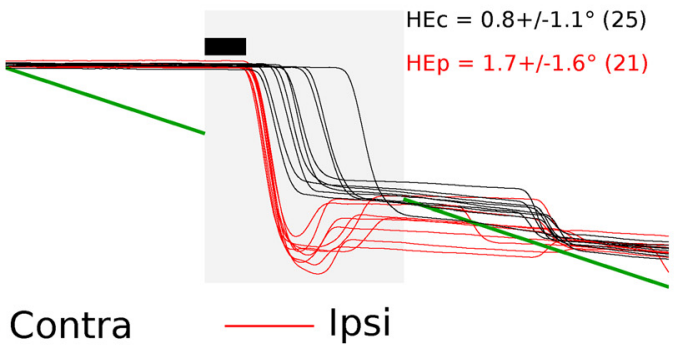

$\mathrm{F}$
VERTICAL

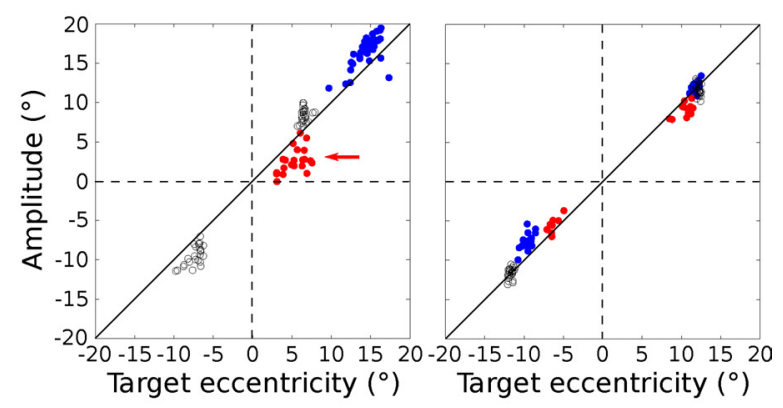

- Ipsi

Figure 3. Time course of horizontal eye movements after target motion onset. Same experiment as in Figures 1, A and B, and 2. A, B, D, E, Green, Target paths; black, control saccades; blue, saccades perturbed by a change in eye position in a direction contralateral to the target motion (Contra); red, the microstimulation moves the eye in the ipsilateral direction (Ipsi). Gray zones delimit the blank interval. Values in each plot correspond to the median \pm interquartile range of horizontal final errors for control (HEC) and perturbed (HEp) saccades (see Materials and Methods). Target velocities were $20 \% \mathrm{~s}(\boldsymbol{A}-\boldsymbol{C})$ and $33 \% \mathrm{~s}(\boldsymbol{D}-\boldsymbol{F})$. Black rectangles represent the stimulation train. $\boldsymbol{C}, \boldsymbol{F}$, The horizontal and vertical amplitude of correction saccades is plotted as a function of the horizontal and vertical target eccentricity at the onset of the correction saccade (amplitude of ideal correction saccade). Blue and red dots correspond to correction saccades after a contralateral and ipsilateral perturbation, respectively. Black dots correspond to unperturbed saccades.

no delay (in-flight corrections) or after a short intersaccadic interval. Figure 3, $C$ and $F$, shows for the same experiments how the horizontal and vertical amplitudes of correction saccades relate, on a trialby-trial basis, to the horizontal amplitude of ideal compensatory saccades, i.e., to the horizontal and vertical eccentricity of the target just before the onset of the correction. The values obtained during the unperturbed trials are also documented for comparison. During contralateral perturbations, the horizontal amplitude of correction saccades $(y)$ increased with target eccentricity $(x)$ along a slope close to unity (orthogonal regression: $y=1.1 x-1.2$, Bravais-Pearson correlation coefficient $r=0.79, F=25.98, p=0.0001, N=18$ for saccades toward the $20^{\circ}$ s target; $y=1.2 x-1.2, r=0.83, F=74.66$, $p=0.00001, N=34$ for those toward the $33 \%$ s target), suggesting that a dynamic, on-line updating of target position is performed to guide the correction saccade. During ipsilateral perturbations, the situation is slightly different because the correction saccades often land on positions located ahead of the target; they are not long enough to orient gaze back on the target location. The horizontal amplitude of correction saccades is smaller than the amplitude observed during unperturbed trials (Fig. 3C,F). For matched values of horizontal target eccentricity [mean $\pm \mathrm{SD}=4.6 \pm 0.7^{\circ}$ (Fig. $3 C$ ) and $6.7 \pm 0.4^{\circ}$ (Fig. $\left.F\right)$, the horizontal amplitude of correction saccades $\left(3.3 \pm 1.0^{\circ}, n=18\right.$ and $3.2 \pm 1.7^{\circ}, n=9$, respectively) is smaller than the horizontal amplitude of unperturbed saccades $\left(4.6 \pm 1.1^{\circ}, n=\right.$ 19 and $8.3 \pm 0.8^{\circ}, n=26$, respectively). Note also that the vertical amplitude of correction saccades matches relatively well with the vertical target eccentricity (Fig. 3C,F, right graphs).

Figure 4 shows observations made during two other experiments [experiment M4 (Fig. 4A,B); experiment M6 (Fig. 4C,D)] where the saccade vectors encoded at the stimulated sites had a vertical component $\left(3.8^{\circ}\right.$ and $10.6^{\circ}$ upward, respectively) larger than the horizontal one $\left(1.2^{\circ}\right.$ and $4.2^{\circ}$ leftward, respectively). In both experiments, the microstimulation evoked a change in vertical eye position (Fig. 4, blue and red arrows), which was fol- 
lowed by a correction saccade toward the target location. During contralateral perturbations (Fig. $4 A, C$, blue traces), the changes in horizontal (median values $=$ -1.3 and $\left.0.2^{\circ}\right)$ and vertical $\left(-1.2^{\circ}\right.$ and $\left.-0.3^{\circ}\right)$ accuracy were again very small despite the relatively large sizes of perturbation [median \pm interquartile range $=$ $0.4 \pm 0.4^{\circ}$ horizontally and $4.5 \pm 0.6^{\circ}$ vertically (Fig. $4 A$ ); $0.5 \pm 2.7^{\circ}$ horizontally and $2.7 \pm 4.7^{\circ}$ vertically (Fig. $4 C$ ) ] and longer flight durations $(92 \pm 29$ and $92 \pm$ $24 \mathrm{~ms}$ ). During ipsilateral perturbations (Fig. $4 B, D$, red traces), the changes in horizontal $\left(-0.2\right.$ and $1.0^{\circ}$, respectively) and vertical $\left(-1.2\right.$ and $-1.0^{\circ}$, respectively) accuracy were also very small in comparison with the sizes of perturbation $\left[-2.3 \pm 0.5^{\circ}\right.$ horizontally and $6.1 \pm 0.5^{\circ}$ vertically (Fig. $4 B$ ); $-6.1 \pm 1.2^{\circ}$ horizontally and $2.7 \pm 5.6^{\circ}$ vertically (Fig. $4 D$ ) ] and the longer flight durations $(101 \pm 23$ and $93 \pm 12 \mathrm{~ms}$ ). Note again that the correction saccades are made either inflight or after an intersaccadic interval.

Figure 5 describes the changes in horizontal (Fig. 5A) and vertical (Fig. $5 B$ ) accuracy when the perturbation is contralateral (black) or ipsilateral (gray) to the target motion for all experiments performed in the two monkeys. In Figure $5 A$, the cumulative distributions show that most correction saccades fall short of the target (negative values) after a contralateral perturbation and ahead of the target (positive values) after an ipsilateral one. The changes in horizontal accuracy are also more variable in the latter case (range, $-1.0-6.4^{\circ}$ ) than in the former case (range, $-3.2-0.9^{\circ}, F$ test, $p<0.05$ ). When one considers the changes in vertical accuracy (Fig. 5B), no significant difference was found between the ipsilateral and contralateral perturbation conditions ( $F$ test, $p>0.05$ ). Because of their different effects on the horizontal accuracy of saccades, we describe separately the performances observed after a contralateral and ipsilateral perturbation.

\section{Accuracy after a contralateral perturbation}

When the direction of the perturbation was contralateral to the target motion, the horizontal accuracies of perturbed and unperturbed saccades were not significantly different (Mann-Whitney test, $p>0.05$ ) from each other in a large number of experiments (55\%). These first observations illustrate an ability to keep track of the moving target location, even when its interception is unexpectedly perturbed. Figure $6 \mathrm{~A}$ shows the changes in horizontal accuracy as a function of the delays that the perturbations added to the time to intercept the moving target (added delay; left graph). If the oculomotor system did not take into account the motion occurring after the onset of the perturbation, Superior colliculus.

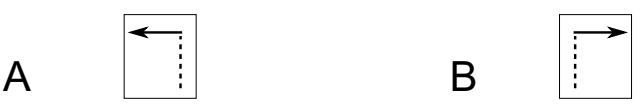

Horizontal component (motion-related)

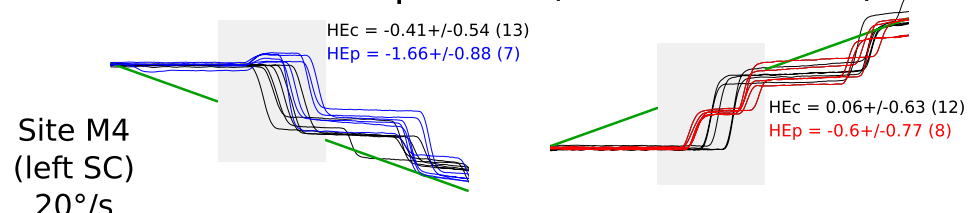

Vertical component (step-related)
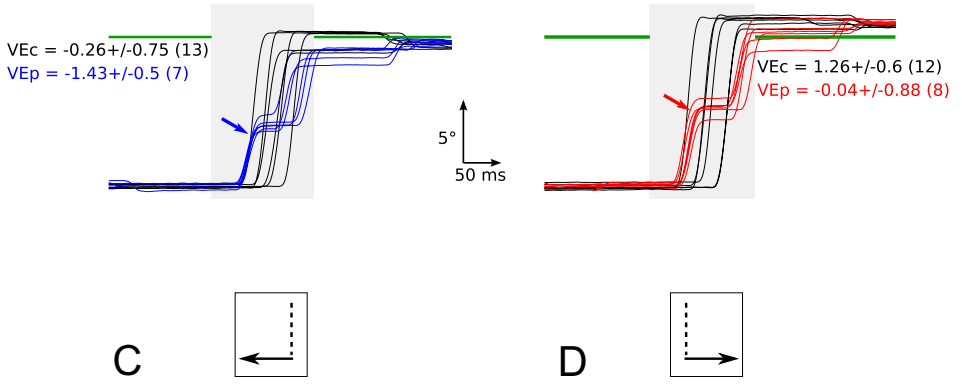

Horizontal component (motion-related)

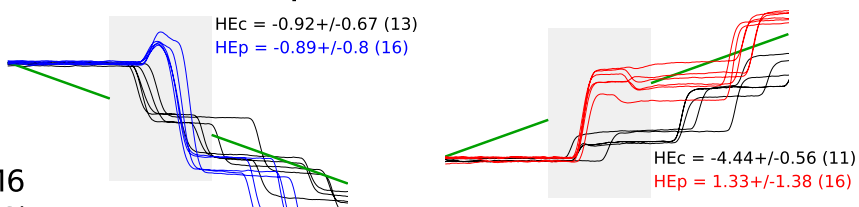

Vertical component (step-related)

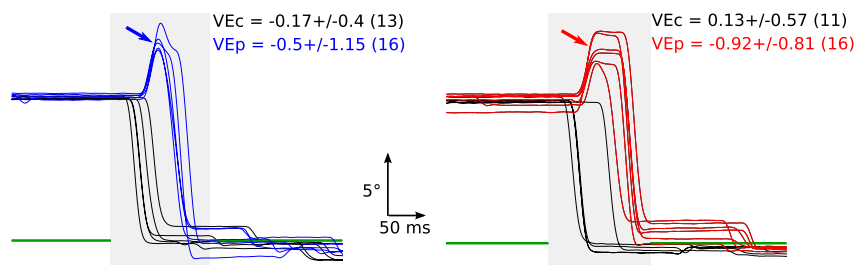

Target Control

Ipsi

Figure 4. Time course of horizontal and vertical eye position for two other experiments where the stimulated site evokes saccades with a larger vertical component. A, B, Experiment M4 (20\%s target velocity). C, D, Experiment M6 ( $\left.33^{\circ} / \mathrm{s}\right)$. Green, Target paths; black, control saccades; blue, saccades perturbed by a contralateral change in eye position (Contra); red, ipsilateral perturbation (Ipsi). Gray zones indicate the blank interval. Values in each plot correspond to the median \pm interquartile range of horizontal and vertical final errors for control (HEC and VEc, respectively) and perturbed (HEp and VEp, respectively) saccades. SC,

then the saccades would be expected to fall short of the target with a horizontal error that increases along a hypothetical slope of -0.02 (when the target moves at $20 \%$ s) or $-0.033(33 \%$ s). For the experiments where the $20 \% \mathrm{~s}$ target velocity was used, the changes in horizontal accuracy $\left(A_{h}\right)$ slightly increased with longer delays $(D)$ without reaching statistical significance $(p>0.05)$ (orthogonal regression: $\mathrm{A}_{\mathrm{h}}=-0.011 \mathrm{D}-0.3, r=-0.26, F=3.13, p=$ $0.08)$. For the other experiments $\left(33^{\circ} / \mathrm{s}\right)$, the correlation was not statistically significant either (orthogonal regression: $A_{h}=$ $-0.006 D-0.3, r=-0.11, F=0.14, p=0.72)$. In both cases, the hypothetical slopes were situated outside the $95 \%$ confidence intervals of the regression slope, indicating that the motion occurring after the onset of perturbations was partly or completely taken into account to generate the correction saccades. In fact, 
A

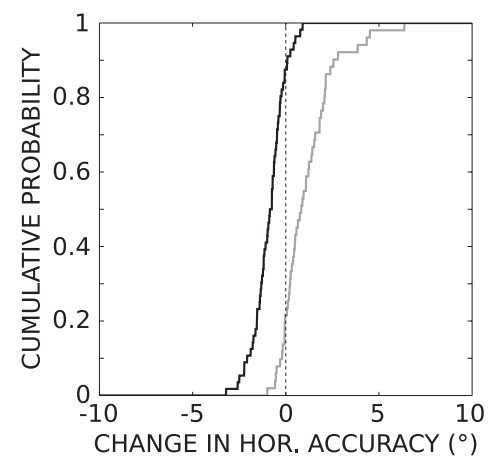

B

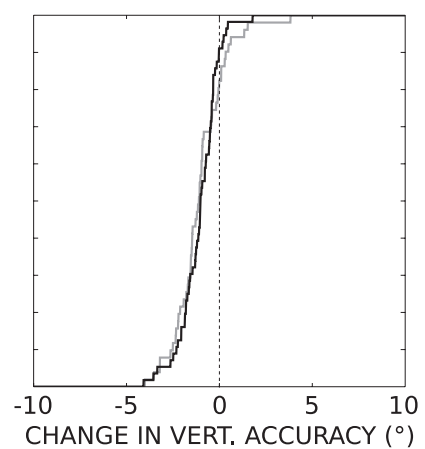

Contralateral Perturbation

_ Ipsilateral Perturbation

Figure 5. Changes in horizontal (HOR.) and vertical (VERT.) accuracy. $\boldsymbol{A}$, Cumulative probability distributions of changes in horizontal accuracy when the perturbation is contralateral (black) or ipsilateral (gray) to the target motion. B, Cumulative distributions of changes in vertical accuracy.

the latter land on horizontal positions that are located ahead $\left(0.8 \pm 2.0^{\circ}\right.$ and $4.3 \pm 1.9^{\circ}$ ahead for the 20 and $33^{\circ} / \mathrm{s}$ targets, respectively) of those expected if the target motion after the perturbation was not taken into account. On average, the horizontal accuracy of correction saccades was slightly hypometric compared with unperturbed saccades (undershoot $=-1.0 \pm 0.8^{\circ}$ and $-0.7 \pm$ $0.8^{\circ}$ for the 20 and $33 \%$ s target, respectively). The lack of difference in horizontal accuracy between the two target velocities (Kolmogorov-Smirnov test, $p=0.73$ ) was an additional result that indicated that the target velocity was taken into account. The hypometria did not depend on the horizontal $\left(P_{\mathrm{h}}\right.$; Fig. $6 \mathrm{~A}$, middle) and vertical $\left(P_{\mathrm{v}}\right.$; Fig. $6 A$, right $)$ amplitude of perturbations (orthogonal regression: $\mathrm{A}_{\mathrm{h}}=-0.04 P_{\mathrm{h}}-0.6, r=0.20, F=2.16$, $p=0.15$ and $\left.\mathrm{A}_{\mathrm{h}}=0.04 P_{\mathrm{v}}-1.0, r=0.14, F=1.03, p=0.32\right)$. Concerning the vertical component (Fig. 6B), its accuracy was not significantly different (Mann-Whitney test, $p>0.05$ ) between perturbed and unperturbed saccades in $56 \%$ of experiments. The changes in vertical accuracy $\left(A_{v}\right)$ were correlated neither with the delays added by the perturbations $\left(20 \% \mathrm{~s}: \mathrm{A}_{\mathrm{v}}=\right.$ $-0.004 D-0.9, r=-0.07, F=0.2, p=0.66 ; 33 \%$ s: $A_{\mathrm{v}}=$ $-0.002 D-0.6, r=-0.05, F=0.03, p=0.87)$ nor with the changes in horizontal eye position $\left(\mathrm{A}_{\mathrm{v}}=-0.04 P_{\mathrm{h}}-0.8, r=\right.$ $-0.17, F=1.65, p=0.21)$. A significant correlation is found between the changes in vertical accuracy and the vertical amplitudes of perturbations $\left(A_{\mathrm{v}}=0.1 P_{\mathrm{v}}-1.5, r=0.30, F=5.6, p=\right.$ 0.02 ) but the relation is such that larger vertical perturbations are associated with a smaller vertical dysmetria.

\section{Accuracy after an ipsilateral perturbation}

When the direction of the perturbation was ipsilateral to the target motion, the horizontal accuracy was not significantly different (Mann-Whitney test, $p>0.05$ ) from that of unperturbed saccades in $43 \%$ experiments. Figure $7 A$ shows the changes in horizontal accuracy as a function of the delays added by the perturbations (left), and the horizontal (middle) and vertical (right) amplitudes of perturbations. A regression analysis suggests larger errors with shorter delays $\left(A_{\mathrm{h}}=-0.026 D+2.8, r=-0.38, F=\right.$ $8.13, p=0.0064)$, although such a relationship accounts for only $14 \%$ of the variance. The better performance with longer delays argues against degradation in the estimate of target position during perturbed trials. Other orthogonal regression analyses re-

vealed a relationship between the changes in horizontal accuracy and the horizontal $\left(A_{\mathrm{h}}=0.1 P_{\mathrm{h}}+0.3, r=0.31, F=5.49, p=\right.$ $0.023)$ and vertical $\left(A_{\mathrm{h}}=0.2 P_{\mathrm{v}}+0.2, r=\right.$ $0.43, F=11.28, p=0.001)$ amplitudes of perturbations. However, such relations account for only $10 \%$ and $19 \%$ of the variance, respectively. In summary, the changes in horizontal accuracy cannot be fully explained by increased delays or by the amplitudes of perturbations. Concerning the vertical component of saccades (Fig. 7B), its accuracy was not significantly different (Mann-Whitney test, $p>0.05$ ) between perturbed and unperturbed saccades in $51 \%$ of experiments. The changes in vertical accuracy were neither correlated with the delays added by the perturbations $\left(A_{\mathrm{v}}=\right.$ $0.011 D-1.7, r=0.16, F=1.24, p=$ $0.27)$ nor with the horizontal changes in eye position $\left(A_{\mathrm{v}}=-0.018 D-0.9, r=\right.$ $-0.06, F=0.17, p=0.68)$. They are correlated with the vertical amplitudes of perturbations $\left(A_{\mathrm{v}}=0.18 P_{\mathrm{v}}-1.7, r=0.35, F=\right.$ $6.87, p=0.012)$ : larger vertical perturbations are associated with a smaller vertical dysmetria.

Because we used short stimulation trains (30-45 ms), the same amplitude of eye displacement could be evoked from different sites in the caudal dSC (Stanford et al., 1996). Therefore, we also analyzed the relationship between the changes in accuracy and the rostrocaudal sites of stimulation. Interestingly, a significant correlation is found between the change in horizontal accuracy and the horizontal component $\left(S_{\mathrm{h}}\right)$ of saccade vectors encoded at the stimulated sites $\left(A_{\mathrm{h}}=0.08 S_{\mathrm{h}}+0.3, r=0.38, F=\right.$ $7.39, p=0.009$; Fig. $8 A$, left). This relationship accounts for only $14 \%$ of the variance. Concerning the vertical component $\left(S_{\mathrm{v}}\right)$ of vectors (Fig. $8 \mathrm{~A}$, right), no significant correlation was found that would suggest an influence on the change in horizontal accuracy $\left(A_{\mathrm{h}}=-0.02 S_{\mathrm{v}}+1.1, r=-0.05, F=0.1, p=0.75\right)$, even though larger changes in accuracy tended to occur when the stimulation was applied in sites encoding targets located along the horizontal meridian (vertical component amplitude $<5^{\circ}$ ). When we analyzed the changes in vertical accuracy (Fig. $8 \mathrm{~B}$ ), we found a significant correlation that suggests an influence of the horizontal $\left(A_{\mathrm{v}}=-0.06 S_{\mathrm{h}}-0.5, r=-0.31, F=4.55, p=0.038\right.$; Fig. $8 B$, left $)$ but not the vertical $\left(A_{\mathrm{v}}=-0.003 S_{\mathrm{v}}-1.1, r=0.01, F=\right.$ $0.0026, p=0.96$; Fig. $8 B$, right) component of saccade vectors encoded at the stimulated sites.

\section{Discussion}

The main finding of our study is that the brain is able to compensate for changes in eye position and delays that are experimentally added before a target moving in the visual periphery is foveated by an interceptive saccade. Such ability suggests the existence of neural mechanisms that continuously encode the ongoing spatiotemporal coordinates of a moving target for guiding its saccadic foveation (Fig. 1C) (Barborica and Ferrera, 2004; Ferrera and Barborica, 2010). A second finding is that an electrical stimulation in the dSC can interfere with this spatiotemporal encoding when it is applied in the colliculus that encodes the visual field traversed by the moving target. Indeed, inaccuracies are sometimes observed in the saccade endpoints when the microstimulation is applied more 


\section{CONTRALATERAL PERTURBATION}
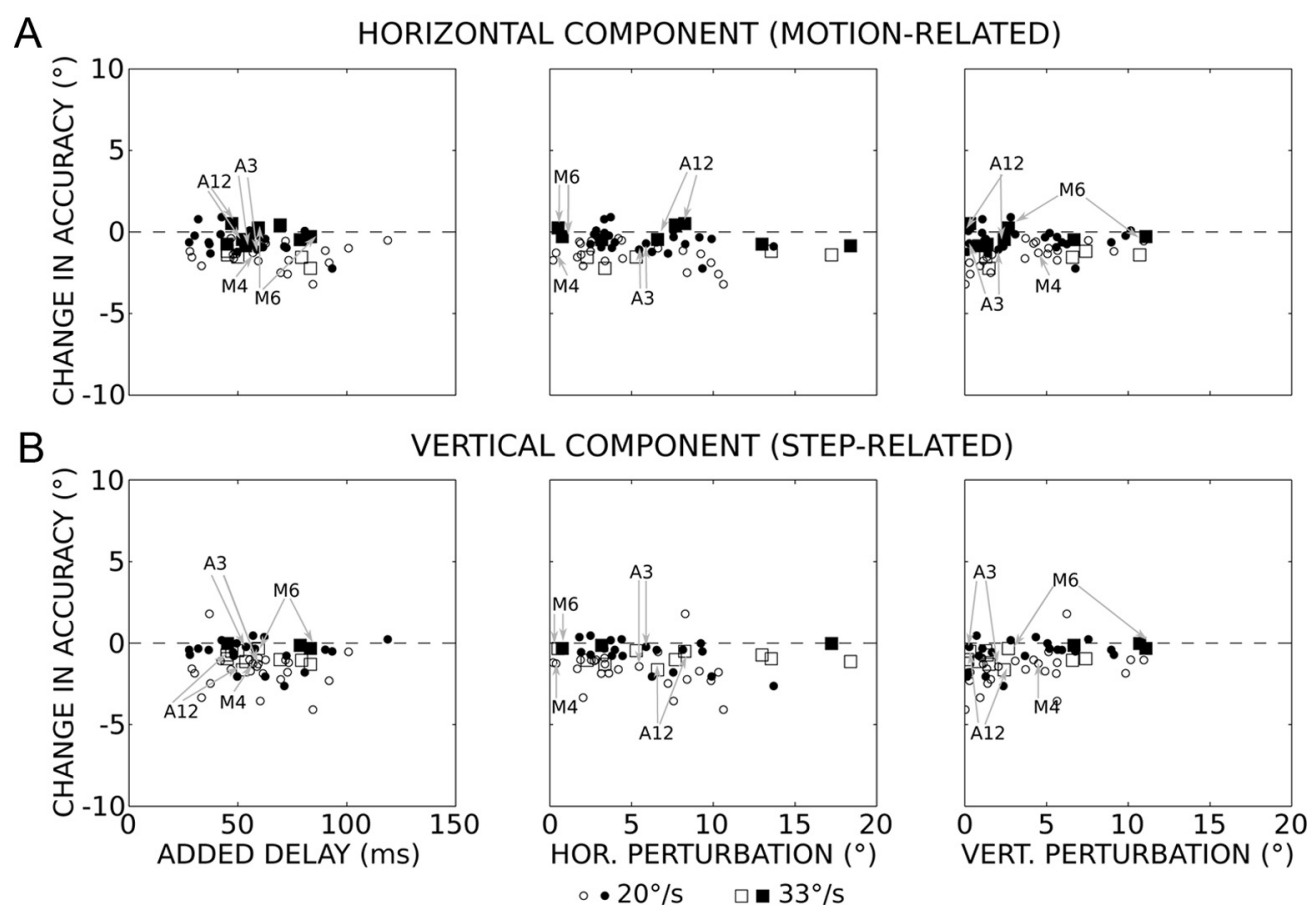

Figure 6. Temporal and spatial perturbations and saccade accuracy when the direction of perturbation is contralateral to the target motion. $A$, Change in horizontal (HOR.) accuracy as a function of the delay added by the perturbation (left), and the horizontal (middle) and vertical (right) amplitudes of perturbations (median values). During unperturbed trials, the flight duration was $34 \pm$ $8 \mathrm{~ms}(n=1176)$. $B$, Change in vertical (VERT.) accuracy. Circles and squares correspond to results obtained with the $20^{\circ} / \mathrm{s}$ and $33^{\circ} / \mathrm{s}$ target velocity, respectively. Open symbols, $p<0.05$; filled symbols, $p>0.05$ (Mann-Whitney test). $N=56$ values (insufficient number of data for 12 sites; see Materials and Methods). The gray arrows indicate the values obtained for the experiments shown in Figures $1, A$ and $B, 2,3$ (sites $A 3$ and A12) and 4 (sites M4 and M6).

caudally in the dSC. Below, we discuss how these findings lead to reconsider the functional scheme that is commonly proposed to explain how the brain generates saccades toward a moving target (Optican, 2009).

Previous studies have tested the robustness of gaze saccades toward a static target and shown that the orienting system compensates for changes in gaze position induced by a brief electrical stimulation within oculomotor regions like the dSC (Schiller and Sandell, 1983; Sparks and Mays, 1983; Pélisson et al., 1989, 1995; Gandhi and Keller, 1999), the paramedian pontine reticular formation (Sparks et al., 1987), or the nucleus raphe interpositus (Paré and Guitton, 1998; Gandhi and Keller, 1999; Gandhi and Sparks, 2007). The numerous examples of unchanged accuracy between unperturbed and perturbed saccades reported in our study (Figs. 3, 4, 6, filled symbols; 7, filled symbols) extend this robustness to the generation of saccades toward a moving target. They also suggest that the brain is able to continuously estimate and keep track of the location of a moving target until the saccade lands on it.

The scheme commonly used to study saccades made toward a moving target considers that the interceptive saccades are driven by signals conveyed by two independent processing streams onto premotor neurons in the PMRF. The first signal would encode the location where the target is initially detected (snapshot) and the second signal would encode its subsequent displacement on the basis of target motion signals (Keller et al., 1996; Optican, 2009). Yet this dual drive hypothesis raises several problems. The first concerns the timing of the snapshot and thus the onset of the hypothetical process that integrates the target motion signals.
The notion of snapshot is indeed far from being obvious when one considers (1) the divergent projections within the visual system, (2) the distributed encoding of target-related signals and motor commands, (3) the delayed transmissions with diverse conduction speeds, and (4) the multiple relays with diverse integration times (Nowak and Bullier, 1997; Schmolesky et al., 1998). In other words, the anatomo-functional architecture of the visual system leads to the situation where a visual event, even discrete (like a single static spot), undergoes a spatiotemporal blurring (Pellionisz and Llinás, 1982) while the activity it evokes on the retina propagates toward the motor centers and muscles. In fact, the snapshot likely corresponds to activities that are spatially and temporally distributed across several regions in the brain. Under such conditions, the onset of the complementary process that integrates target motion signals becomes undefined as well. The second problem concerns the discrete nature of the target position sampling (Barmack, 1970; Fleuriet et al., 2011). A discrete sampling is indeed questioned by experiments showing that two saccades made to different goals can be concurrently programmed, even just before launching a saccade (McPeek and Keller, 2001; McPeek et al., 2003; Port and Wurtz, 2003). The last problem concerns the time interval during which target motion signals are integrated to encode the displacement of the target after its position is sampled. Indeed, the numerous cases of accurate interception reported here would imply the existence of mechanisms in the brain that estimate delays that can be prolonged in a variable manner to extend the integration interval until the end of saccades. Moreover, this chronometric control would have to be maintained even when a pause separates the end 


\section{IPSILATERAL PERTURBATION}

\section{A \\ HORIZONTAL COMPONENT (MOTION-RELATED)}
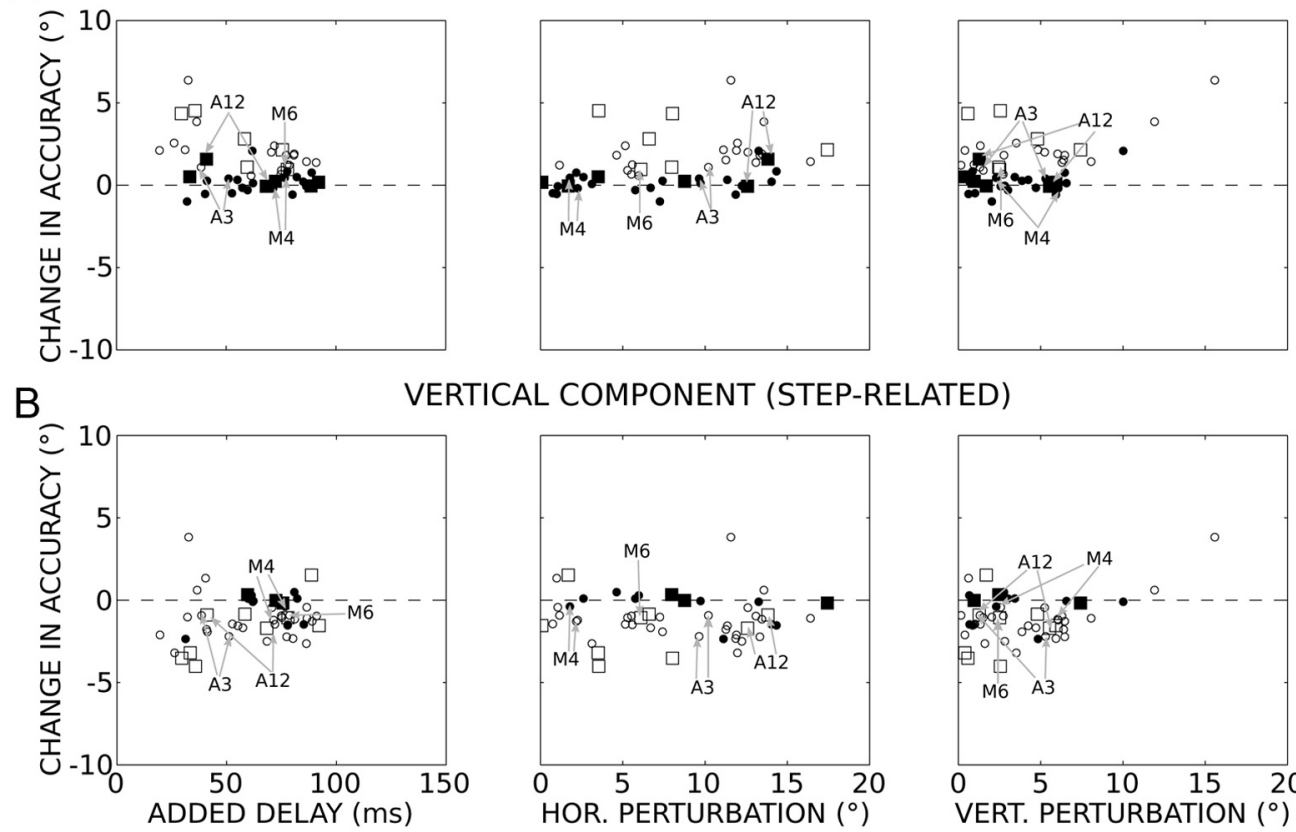

ERTICAL COMPONENT (STEP-RELATED)
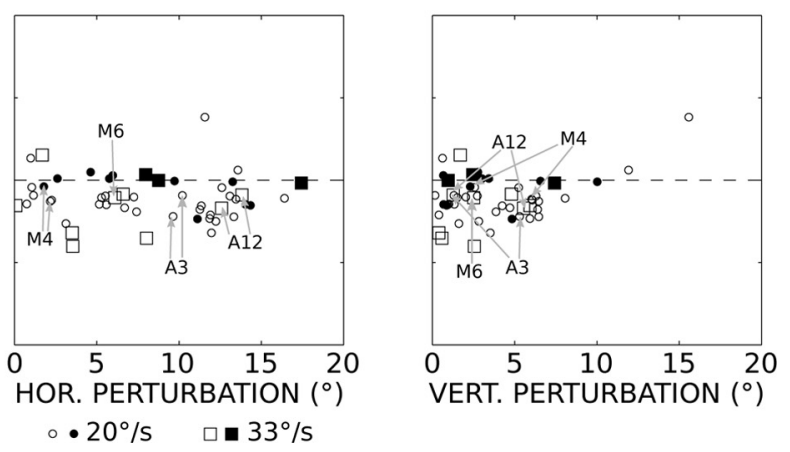

Figure 7. Temporal and spatial perturbations and saccade accuracy when the direction of perturbation is ipsilateral to the target motion. $\boldsymbol{A}$, Change in horizontal (HOR.) accuracy as a function of the delay added by the perturbation (left), and the horizontal (middle) and the vertical (right) amplitude of perturbations. Note that the flight duration is $34 \pm 8 \mathrm{~ms}$ (median \pm interquartile range) during unperturbed trials $\left(n=1176\right.$ ). $\boldsymbol{B}$, Change in vertical (VERT.) accuracy. Circle and square symbols correspond to results obtained with the $20^{\circ} / \mathrm{s}$ and $33^{\circ} / \mathrm{s}$ target velocity, respectively. 0 pen symbols, $p<0.05$; filled symbols, $p>0.05$ (Mann-Whitney test). $N=51$ values (insufficient number of data for 16 sites, see Materials and Methods). The gray arrows indicate the values obtained for the experiments shown in Figures 1, $A$ and $B, 2,3$ (sites $A 3$ and $A 12$ ), and 4 (sites M4 and M6).

of the stimulation-induced change in eye position from the onset of the correction saccade (Figs. 3, 4), i.e., in the absence of premotor activities. Therefore, instead of considering two separate drive commands, we propose that a single dynamic command encoding the current and expected target location feeds the same feedback mechanism as the one proposed for the guidance of saccades toward a static target (Van Gisbergen et al., 1981; Guitton, 1992; Robinson, 1992; Sparks, 2002; Goffart, 2009). This alternative hypothesis does not assume any chronometric mechanism for controlling the end of saccades (Thier et al., 2000); the premotor neurons in the PMRF keep bursting until the estimate of the eye displacement (or position) matches with the expected target displacement (or position).

Our study shows that the accuracy of saccades can be affected when the microstimulation is applied in the dSC, which is supposed to relay the hypothetical snapshot. More specifically, overshoots are observed when the target moves in the visual field that is contralateral to the stimulated site (i.e., when the perturbation is ipsilateral to the target motion direction). This overshoot increases with the horizontal amplitude of the vector encoded at the stimulated site (Fig. 8). It could be argued that the electrical microstimulation generates an activity in the dSC that interferes with signals encoding the snapshot or the stimulation-induced change in eye position. However, these possibilities are not consistent with results from other studies that tested the compensation abilities with static targets in monkeys and did not report comparable inaccuracies (Schiller and Sandell, 1983; Sparks and Mays, 1983). There are two possible explanations that could account for the inaccuracy sometimes observed during ipsilateral perturba- tions. One, the dSC microstimulation could generate activities that interfere with the processing of target motion signals (Nummela and Krauzlis, 2011). Two, the microstimulation could interfere with signals encoding the expected target location. Indeed, if target motion signals from frontal eye field (Barborica and Ferrera, 2003; Cassanello et al., 2008; Ferrera and Barborica, 2010) or medial superior temporal (MST) area (Maioli et al., 1992) influence the population activity in the dSC for encoding the expected target location (Witten et al., 2006), microstimulation could affect either a propagation of activity in the dSC (Arbib and Lara, 1982) or the recruitment of additional neurons that would burst before the interceptive saccade is launched toward the target (for the remapping hypothesis, see Fleuriet et al., 2011).

In conclusion, the results reported here bring new and difficult constraints to the dual-drive hypothesis by showing that the interception can remain accurate when a delay and change in eye position are experimentally introduced before saccade onset. Instead of considering the existence of a clock in the brain that estimates delays for reaching a goal, we propose that the brain builds an estimate of the expected and current spatiotemporal (hic et nunc) coordinates of the target, an estimate that remains relatively independent of the time taken to react and to foveate it. Thus, a single dynamic command encoding the expected target location would feed the same local feedback mechanism as proposed for the guidance of saccades toward a static target. Further research is required to determine how internal models of moving targets are built to ultimately conform to the ongoing behavior and whether a similar functional organization underlies the control of other types of goal-directed actions (Jeannerod, 1997). 


\section{IPSILATERAL PERTURBATION}

\section{A HORIZONTAL COMPONENT (MOTION-RELATED)}
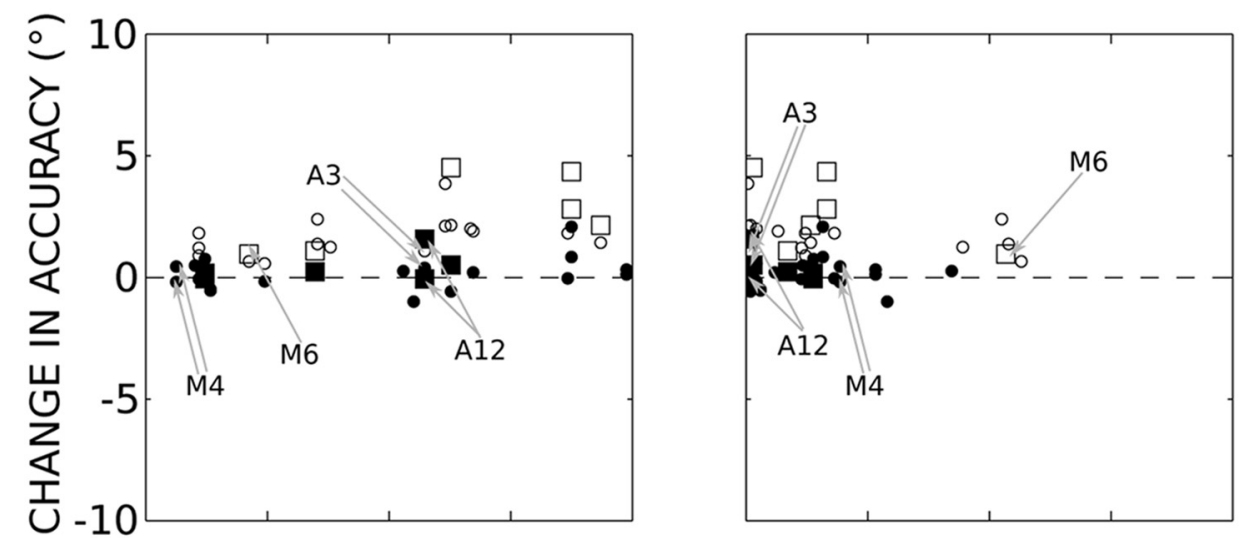

B
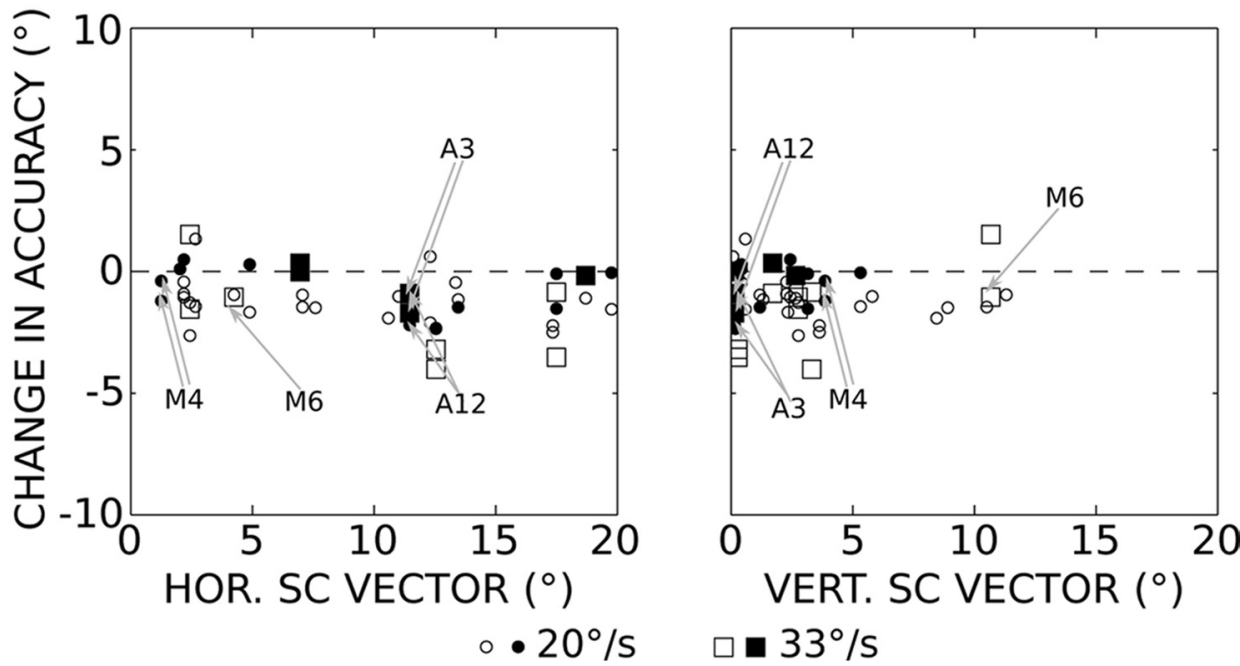

Figure 8. Vector encoded at the stimulation sites and saccade accuracy when the perturbation is ipsilateral to the target motion. $\boldsymbol{A}$, Change in horizontal (HOR.) accuracy as a function of the horizontal (left) and vertical (right) component of the dSC vector (median values). $\boldsymbol{B}$, Change in vertical (VERT.) accuracy. Circle and square symbols correspond to results obtained with the $20^{\circ} / \mathrm{s}$ and $33^{\circ} / \mathrm{s}$ target velocity, respectively. Open symbols, $p<0.05$; filled symbols, $p>0.05$ (Mann-Whitney test). $N=47$ values (data from 31 of 34 sites; see Materials and Methods). The gray arrows indicate the values obtained for the experiments shown in Figures $1, A$ and $B, 2,3$ (sites $A 3$ and $A 12$ ), and 4 (sites M4 and M6).

\section{References}

Arbib MA, Lara R (1982) A neural model of the interaction of tectal columns in prey-catching behavior. Biol Cybern 44:185-196.

Barborica A, Ferrera VP (2003) Estimating invisible target speed from neuronal activity in monkey frontal eye field. Nat Neurosci 6:66-74.

Barborica A, Ferrera VP (2004) Modification of saccades evoked by stimulation of frontal eye field during invisible target tracking. J Neurosci 24:3260-3267.

Barmack NH (1970) Modifications of eye movements by instantaneous changes in the velocity of visual targets. Vision Res 10:1431-1441.

Cassanello CR, Nihalani AT, Ferrera VP (2008) Neuronal responses to moving targets in monkey frontal eye fields. J Neurophysiol 100:1544-1556.

Ferrera VP, Barborica A (2010) Internally generated error signals in monkey frontal eye field during an inferred motion task. J Neurosci 30:11612-11623.

Fleuriet J, Hugues S, Perrinet L, Goffart L (2011) Saccadic foveation of a moving visual target in the rhesus monkey. J Neurophysiol 105:883-895.

Fuchs AF, Robinson DA (1966) A method for measuring horizontal and vertical eye movement chronically in the monkey. J Appl Physiol 21:1068-1070.

Gandhi NJ, Katnani HA (2011) Motor functions of the superior colliculus. Annu Rev Neurosci 34:205-231.
Gandhi NJ, Keller EL (1999) Comparison of saccades perturbed by stimulation of the rostral superior colliculus, the caudal superior colliculus, and the omnipause neuron region. J Neurophysiol 82:3236-3253.

Gandhi NJ, Sparks DL (2007) Dissociation of eye and head components of gaze shifts by stimulation of the omnipause neuron region. J Neurophysiol 98:360-373.

Gellman RS, Carl JR (1991) Motion processing for saccadic eye movements in humans. Exp Brain Res 84:660-667.

Goffart L (2009) Saccadic eye movements. In: Encyclopedia of neuroscience (Squire LR, ed.), pp. 437-444. Oxford: Academic.

Guan Y, Eggert T, Bayer O, Büttner U (2005) Saccades to stationary and moving targets differ in the monkey. Exp Brain Res 161:220-232.

Guitton D (1992) Control of eye-head coordination during orienting gaze shifts. Trends Neurosci 15:174-179.

Hall WE, Moschovakis AK (2004) The superior colliculus: new approaches for studying sensorimotor integration. Boca Raton, FL: CRC.

Jeannerod M (1997) The cognitive neuroscience of action. Oxford: Wiley-Blackwell.

Judge SJ, Richmond BJ, Chu FC (1980) Implantation of magnetic search 
coils for measurement of eye position: an improved method. Vision Res 20:535-538.

Keller E, Johnsen SD (1990) Velocity prediction in corrective saccades during smooth-pursuit eye movements in monkey. Exp Brain Res 80:525-531.

Keller EL, Gandhi NJ, Weir PT (1996) Discharge of superior collicular neurons during saccades made to moving targets. J Neurophysiol 76:3573-3577.

Maioli MG, Domeniconi R, Squatrito S, Riva Sanseverino E (1992) Projections from cortical visual areas of the superior temporal sulcus to the superior colliculus in macaque monkeys. Arch Ital Biol 130:157-166.

Mays LE, Sparks DL (1980) Dissociation of visual and saccade-related responses in superior colliculus neurons. J Neurophysiol 43:207-232.

McPeek RM, Keller EL (2001) Short-term priming, concurrent processing, and saccade curvature during a target selection task in the monkey. Vision Res 41:785-800.

McPeek RM, Han JH, Keller EL (2003) Competition between saccade goals in the superior colliculus produces saccade curvature. J Neurophysiol 89:2577-2590.

Nowak LG, Bullier J (1997) The timing of information transfer in the visual system. In: Cerebral cortex: extrastriate cortex in primates (Rockland $\mathrm{K}$, Kaas JH, Peters A, eds.). New York: Plenum.

Nummela SU, Krauzlis RJ (2011) Superior colliculus inactivation alters the weighted integration of visual stimuli. J Neurosci 31:8059-8066.

Optican LM (2009) Oculomotor system: models. In: Encyclopedia of neuroscience (Squire LR, ed.), pp. 25-34. Oxford: Academic.

Paré M, Guitton D (1998) Brain stem omnipause neurons and the control of combined eye-head gaze saccades in the alert cat. J Neurophysiol 79:3060-3076.

Pélisson D, Guitton D, Munoz DP (1989) Compensatory eye and head movements generated by the cat following stimulation-induced perturbations in gaze position. Exp Brain Res 78:654-658.

Pélisson D, Guitton D, Goffart L (1995) On-line compensation of gaze shifts perturbed by micro-stimulation of the superior colliculus in the cat with unrestrained head. Exp Brain Res 106:196-204.

Pellionisz A, Llinás R (1982) Space-time representation in the brain: the cerebellum as a predictive space-time metric tensor. Neuroscience 7:2949-2970.
Port NL, Wurtz RH (2003) Sequential activity of simultaneously recorded neurons in the superior colliculus during curved saccades. J Neurophysiol 90:1887-1903.

Robinson DA (1992) Implications of neural networks for how we think about brain function. Behav Brain Sci 15:644-655.

Schiller PH, Sandell JH (1983) Interactions between visually and electrically elicited saccades before and after superior colliculus and frontal eye field ablations in the rhesus monkey. Exp Brain Res 49:381-392.

Schmolesky MT, Wang Y, Hanes DP, Thompson KG, Leutgeb S, Schall JD, Leventhal AG (1998) Signal timing across the macaque visual system. J Neurophysiol 79:3272-3278.

Schreiber C, Missal M, Lefèvre P (2006) Asynchrony between position and motion signals in the saccadic system. J Neurophysiol 95:960-969.

Segraves MA, Goldberg ME, Deng SY, Bruce CJ, Ungerleider LG, Mishkin M (1987) The role of striate cortex in the guidance of eye movements in the monkey. J Neurosci 7:3040-3058.

Sparks DL (2002) The brainstem control of saccadic eye movements. Nat Rev Neurosci 3:952-964.

Sparks DL, Mays LE (1983) Spatial localization of saccade targets. I. Compensation for stimulation-induced perturbations in eye position. J Neurophysiol 49:45-63.

Sparks DL, Mays LE, Porter JD (1987) Eye movements induced by pontine stimulation: interaction with visually triggered saccades. J Neurophysiol 58:300-318.

Stanford TR, Freedman EG, Sparks DL (1996) Site and parameters of microstimulation: evidence for independent effects on the properties of saccades evoked from the primate superior colliculus. J Neurophysiol 76:3360-3381.

Thier P, Dicke PW, Haas R, Barash S (2000) Encoding of movement time by populations of cerebellar Purkinje cells. Nature 405:72-76.

Van Gisbergen JA, Robinson DA, Gielen S (1981) A quantitative analysis of generation of saccadic eye movements by burst neurons. J Neurophysiol 45:417-442.

Witten IB, Bergan JF, Knudsen EI (2006) Dynamic shifts in the owl's auditory space map predict moving sound location. Nat Neurosci 9:1439-1445. 